\title{
A dislocation based material model for warm forming simulation
}

\author{
Journal Article
}

Author(s):

Berisha, Bekim (D); Hora, Pavel; Wahlen, Arne; Tong, Longchang

Publication date:

2008

Permanent link:

https://doi.org/10.3929/ethz-b-000011290

Rights / license:

In Copyright - Non-Commercial Use Permitted

Originally published in:

International Journal of Material Forming 1(3), https://doi.org/10.1007/s12289-008-0367-7 


\title{
A dislocation based material model for warm forming simulation
}

\author{
Bekim Berisha · Pavel Hora • Arne Wahlen • \\ Longchang Tong
}

Received: 31 January 2008 / Accepted: 26 March 2008 / Published online: 5 June 2008

(C) Springer/ESAFORM 2008

\begin{abstract}
Based on experimental results, a dislocation material model describing the influence of dynamic strain aging on the deformation behavior at elevated temperatures is presented. One and two stage loading tests at different temperatures were performed in order to describe the hardening behavior during the deformation at elevated temperatures as well as the hardening behavior after the dynamic strain aging process. Bergström's theory of work hardening was used as a basis for the model development. In the proposed model a relationship between material coefficients of the classical Bergström model and temperature was investigated. The aim of the new material model was to introduce the least possible amount of new parameters as well as to facilitate the mathematical determination of parameters during the fitting of the model with the experimental data. The developed model was implemented in an in-house FE-Code in order to simulate the material behavior due to the dynamic strain aging. Representative simulation results were compared with the experimental data in order to validate the efficiency and the application range of the model. Simulation of the forming process provided data for optimizing strength properties and enabled process control.
\end{abstract}

B. Berisha $(\varangle) \cdot$ P. Hora · A. Wahlen · L. Tong Institute of Virtual Manufacturing, ETH Zurich, Tannenstr. 3, 8092 Zurich, Switzerland

e-mail: berisha@ivp.mavt.ethz.ch
Keywords Dislocation density • Warm forming • Dynamic strain aging $\cdot$ Two stage deformation

\section{Introduction}

The aim of this study was the investigation of the dynamic strain aging behavior of a high strength low carbon steel. Dynamic strain aging is a phenomenon in which the free interstitial atoms diffuse around dislocations and inhibit dislocation motion. As shown in Bergstöm (1983) or later in van Liempt et al. (2002) and Gündüz (2002) the interaction between dislocations and solutes is manifested in increasing the flow stress and a negative strain-rate sensitivity. Moreover, if such a steel is formed at a certain temperature, the strength after cooling to room temperature and reloading is much higher than the strength of the undeformed material. Besides temperature dependence, the dynamic strain aging effect depends on the material composition and especially on the free-nitrogen and/or carbon concentration. It is well recognized that the effect of nitrogen on strain aging is more pronounced as it has a higher solubility and diffusion coefficient in steel. In the early work of Bergstöm (1983), transmission electron microscopy investigations were performed at room temperature to study dislocations and dislocation structures in undeformed as well as in deformed materials. It is important to mention that those studies are very helpful to derive a relationship between the dislocation density and the deformation, but in some cases e.g. at elevated temperatures it is very difficult to do such studies and to make quantitative statements regarding the physical phenomena. 


\section{Dislocation based material modeling}

The theory of plasticity has been intensively developed in the last decades. Most of the publications deal with phenomenological aspects of the hardening in metals. Only a small range of material models are based on micro mechanical phenomena. Work hardening is caused by accumulation of dislocations. Therefore the flow stress is a function of the total dislocation density. In the well established stress-strain relationship (Eq. 1) the total dislocation density $\rho$ is a function of the equivalent plastic strain $\epsilon$ (Mecking and Kocks 1981).

$\sigma=\sigma_{0}+c G \sqrt{\rho(\epsilon)}$

where $\sigma_{0}$ is the strain independent yield stress, $c$ is a constant which contains information about the Burgers vector and other crystal properties and $G$ is the shear modulus. The major problem on formulating a dislocation theory is to derive the relationship between the dislocation density and the deformation. The dislocation theory of Bergstöm (1970) is based on the average behavior of a large number of dislocations, mobile as well as immobile dislocations. The different types of dislocations are used to understand more precisely the physical phenomena like creation, immobilization, remobilization and annihilation of dislocations and to derive a relationship between the dislocation density and the plastic strain, but they are not explicitly included in the final model. Bergström proposed the following evolution equation of the dislocation density $\rho$ :

$\frac{\partial \rho}{\partial \epsilon}=B-R \rho$

where $B$ is the immobilization rate of dislocations and $R$ is a parameter describing the remobilization of dislocations. Under some assumptions and especially the strain independence of the immobilization rate $B$, Eq. 2 can be integrated analytically, and one obtains a relationship between dislocation density $\rho$ and the plastic strain $\epsilon$.

$\rho(\epsilon)=\frac{B}{R}\left[\left(\rho_{0}-1\right) e^{-R \epsilon}+1\right]$

where $\rho_{0}$ is the initial dislocation density. The major benefit of the physically based material models is that the prediction of the material behavior for deformations larger than the available experimental data is more secured, whereas in the region of measured data there is no significant difference. Furthermore, the physical meaning of the model parameters is very helpful regarding the discussion of the model prediction as well as the real material behavior.

\section{Experiments and the enhanced Bergström model}

In order to simulate the warm forming process and to predict the material properties at room temperature, one stage compression tests of cylindrical samples were performed at a constant strain rate and constant temperature, in a temperature range of $25-500^{\circ} \mathrm{C}$. The experiments were performed on a Bähr DIL 805 dilatometer using cylindrical specimens with a ratio of length to diameter 3:2. In order to reduce friction effects during the deformation, graphite lubrication was used. The temperature of the specimen was controlled with one thermocouple which was spot welded on the specimen.

The experimental data were fitted with the Bergström model Eq. 1. Figure 1 shows the temperature dependence of the material coefficients as well as the approximation functions.
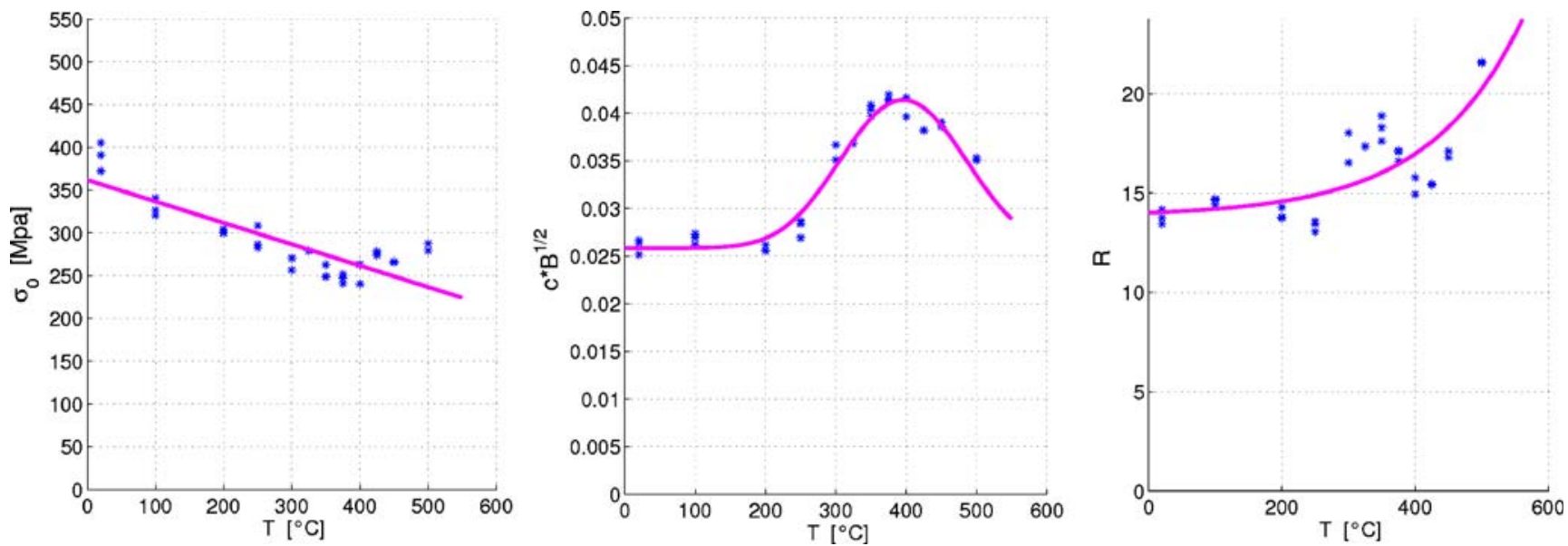

Fig. 1 Temperature dependence of the material coefficients $\sigma_{0}$ : yield stress, $c \sqrt{B}$ : product of the constant $c$ and the immobilization rate $B$ of dislocations, $R$ : remobilization parameter of dislocations 
The temperature dependence of the yield strength has a common trend and was approximated with a linear function

$\sigma_{0}(T)=a-b T$

More complex is the temperature dependence of the parameter $B$, which was described with a Gauss function Eq. 5. The maximum value of the immobilization rate $B(T)$ is achieved when the velocity of the free interstitial atoms is the same as the velocity of the mobile dislocations.

$B(T)=1+\gamma e^{-\left(\frac{T-T_{0}}{\tau}\right)^{2}}$

The parameter $\gamma$ contains information about the concentration of the free interstitial atoms. If the material does not contain interstitial atoms, the parameter $\gamma$ is equal to zero and the immobilization rate $B$ do not describe dynamic strain aging. $T_{0}$ is the temperature at which the immobilization of dislocations achieved its maximum. $\tau$ is the parameter which defines the width of the function $B$ or in other words, $\tau$ defines the temperature range of the dynamic strain aging effect.

The characteristics of the remobilization parameter $R$ are not very pronounced. However, as the remobilization value increases with increasing temperature, $R$ is reasonably approximated with an exponential function Eq. 6.

$R(T)=R_{0}+\alpha e^{-\frac{\beta}{T}}$

where $R_{0}$ is the temperature independent remobilization parameter, $\alpha$ and $\beta$ describe saturation behavior of the remobilization. The temperature dependence of the shear modulus $G$ was not investigated in this work. The following function was fitted with the experimental data which were published in Anon (1990) in order to take the temperature dependence into account

$G(T)=g_{0}+g_{1} T+g_{2} T^{2}$

where $g_{0}, g_{1}$ and $g_{2}$ are constants. The initial dislocation density is normalized by the quotient of $R$ and $B$

$\rho_{0}(T)=\frac{R(T)}{B(T)} \tilde{\rho}$

where $\tilde{\rho}$ is the real initial dislocation density. Since it is assumed to be very small for the undeformed material, it was set to zero. The parameter identification is by no means trivial in the development of the material models. In some cases one has to adapt the equations in order to determine suitable coefficients. For example the constant $c$ of Eq. 1, which contains information about the Burgers vector and other material properties, is multiplied with the square root of $B$ and afterwards the product $c \sqrt{B}$ is fitted to the data, in order to avoid

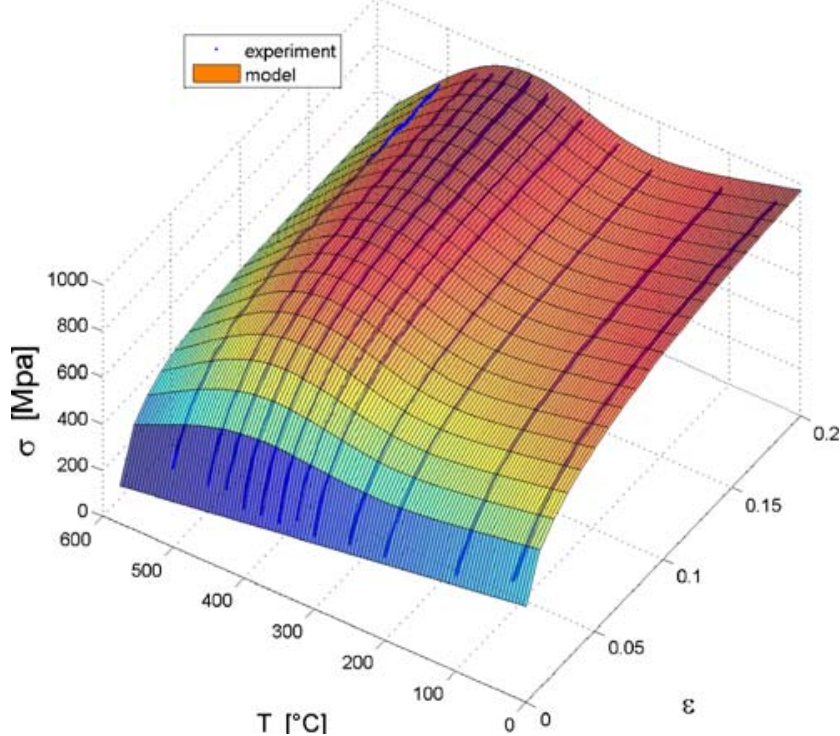

Fig. 2 Yield stress as a function of the plastic strain and temperature

unphysical values of the determined parameters, Fig. 1. The model parameters are determined using a gradient based optimization method. The experimental data of the temperature dependent compression tests and the fit of the data is shown in Fig. 2.

In addition to the temperature dependence of the stress, the temperature dependence of the dislocation density is shown in Fig. 3 which was calculated with

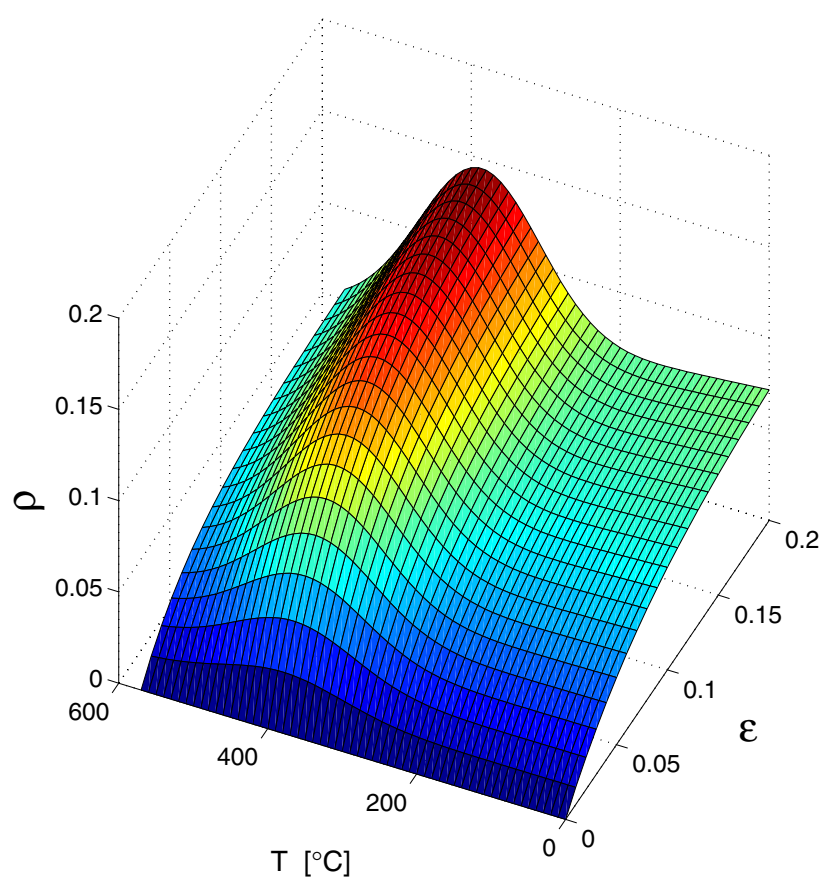

Fig. 3 Dislocation density as a function of the plastic strain and the temperature 
the function Eq. 3, where the parameter $B$ and $R$ are the temperature dependent function Eqs. 5 and 6, respectively.

The effect of the dynamic strain aging is very pronounced and is manifested with an increasing of the dislocation density in a certain temperature range. The temperature dependence of the immobilization rate $B(T)$ is the major component in modeling the behavior of the dislocation density. As discussed before, the function $B(T)$ was fitted with experimental data and this supports the assumption of a temperature dependent dislocation density.

\section{Investigation of the two stage loading}

In order to investigate material properties at room temperature for the warm pre-deformed material, two stage tests were performed. This kind of deformation requires a particular analysis of the measured data during the two deformations. Furthermore, the influence of the pre-deformation on the saturation of the dislocation density and on the hardening during the loading at the second stage was investigated. Cylindrical samples were first compressed at a temperature range of $25-500{ }^{\circ} \mathrm{C}$. The saturation value of the dislocation density is given by

$\rho_{\text {sat }}(T)=\frac{B(T)}{R(T)}$

where $T$ is the temperature of the first or the second deformation, see Fig. 3. If the strain of the first deformation exceeds a certain value, the available dislocation density is higher than the saturation value of the dislocation density at room temperature. Therefore, during the second deformation the model predicts softening. In order to avoid this characteristic of the model, the following assumption is made:

$\rho=\rho_{L}+\rho_{F}$

which leads to a splitting of the total dislocations into locked $\rho_{L}$ and free dislocations $\rho_{F}$. Locked dislocations $\rho_{L}$ are those dislocations which have interacted with the free interstitial atoms and free dislocations $\rho_{F}$ are those which did not interact, or in other words they did not capture interstitial atoms. Substituting the dislocation density Eq. 3 into the evolution Eq. 2, the following equation is obtained

$\frac{\partial \rho}{\partial \epsilon}=B\left(1-\rho_{0}\right) e^{-R \epsilon}$

As long as one stage processes are modeled, the evolution equation of the dislocation density Eq. 11 is equal

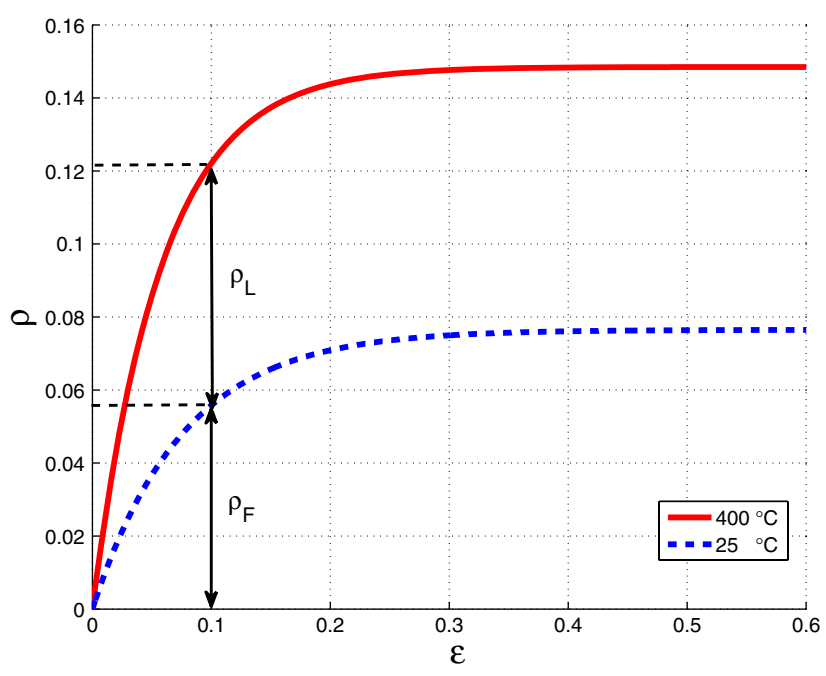

Fig. 4 Dislocation density at two different temperatures

to the expression Eq. 2. This is explained in Fig. 4. For a certain plastic strain one gets the corresponding dislocation density and vice versa. But if the first deformation is performed at a higher temperature e.g. at $400^{\circ} \mathrm{C}$ and the second deformation is performed at room temperature, the two evolution equations of the dislocation density are no longer equal. Due to the assumption discussed above, Eq. 2 can be rewritten and the following evolution equation for the dislocation density is obtained.

$\frac{\partial \rho}{\partial \epsilon}=B-R\left(\rho-\rho_{L}\right)$

The statement on the assumption Eq. 12 is that the remobilization parameter $R$ is no longer applied to the total dislocation density but only to the free dislocation density. In other words, no remobilization of locked dislocations is possible. Splitting off the total dislocations in diverse dislocation classes is a common assumption and is widely accepted, but the way how this is done is in generally different e.g. Bergstöm (1983), van Liempt et al. (2002). The dislocations which were generated at a higher temperature for example at $400^{\circ} \mathrm{C}$ they would not have been generated at room temperature, therefore the assumption Eq. 10 seems to be a good choice to describe the amount of the available dislocations which can be remobilized.

\section{Strain-rate sensitivity}

Normally, materials show at least a small hardening effect with increasing strain-rate. Materials with dynamic strain aging characteristics show a negative strain-rate sensitivity (Zeghib and Klepaczko 1996), see Fig. 5. 


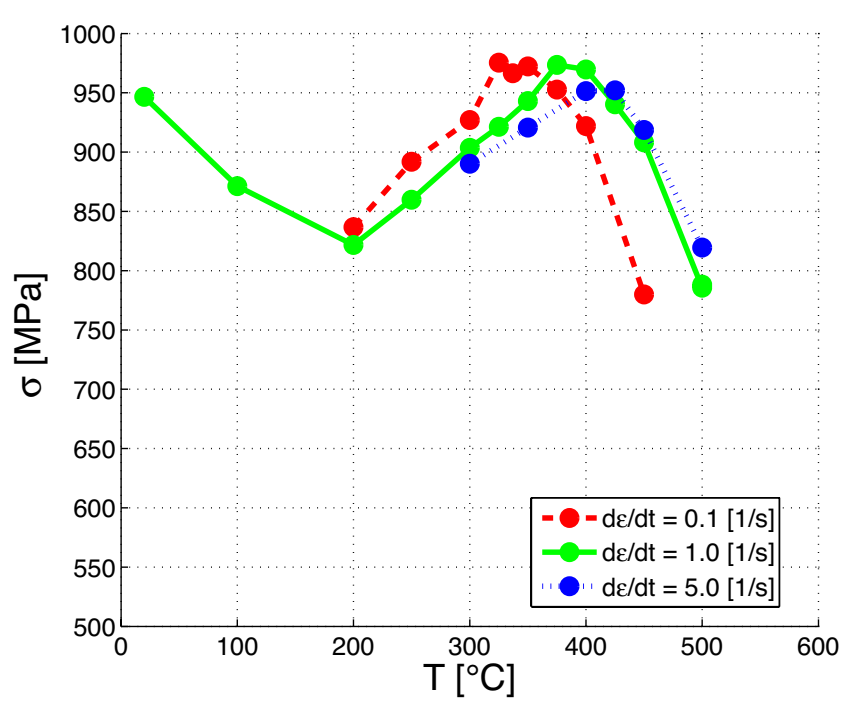

Fig. 5 Negative strain-rate sensitivity of the flow stress at $\epsilon=0.2$

The peak of the stress is shifted to higher temperatures when the strain rate increases, while its peak stress decreases. The reason why this phenomenon happens is because the velocity of the dislocations is very high, and not all solutes can follow them for an interaction (Jiang et al. 2007). For very high strain rates, no dynamic strain aging effect is observed.

Through an Arrhenius expression Eq. 13 which gives the same activation energy as that for the diffusion of carbon in iron, it is possible to calculate the strain rate for the given temperature

$\dot{\epsilon}=C \cdot e^{-\frac{Q}{R_{g} \cdot T}}$

where $C$ is a factor which contains information about the Burgers vector, radius of the Cottrell cloud, dislocation density, vacancy concentration and about diffusion frequency of the solute atoms (Bergstöm 1983; Cottrell 1953). $Q$ is the activation energy and $R_{g}$ is the universal gas constant. For a given strain rate and temperature one can determine the constant $C$. Afterwards one can calculate any other temperature for the corresponding strain rate, for example $\dot{\epsilon}=1 s^{-1}$ and $\dot{\epsilon}=5 s^{-1}$, see Fig. 5. The calculated temperatures coincide very well with the measured temperatures. Furthermore, the calculated temperature can be used in Eq. 5 for the parameter $T_{0}$ in order to take the strain rate dependence into account. This will shift the curve as discussed before.

\section{Discussion}

The new material model approximates the experimental data of the warm compressed specimens accurately, see Fig. 2. The results of the simulation and experimental data of two stage compression tests are shown in Fig. 6. The prediction of the yield strength of the second stage has a high accuracy, independent from the plastic pre-strain of the first deformation. The temperature behavior of the yield strength is similar to the behavior of the immobilization rate $B(T)$. The higher the diffusion of the interstitial atoms into mobile dislocations during the first plastic deformation, the higher the yield strength at room temperature. If the first and the second deformation is done at room temperature, or almost at room temperature, no dynamic strain aging effect is possible.

Figure 7 shows some simulation examples of the two stage compression tests including the hardening behavior of the second deformation at room temperature, where the first deformation was carried out at $T=300^{\circ} \mathrm{C}$ and a strain rate of $\dot{\epsilon}=1 \mathrm{~s}^{-1}$. It is important to emphasize that only the data of the first deformation were fitted and the prediction of the hardening behavior of the second stage is due to the assumption of the dislocation splitting into locked and free dislocations Eq. 10. It is obvious that there is a discontinuous stress behavior due to the dynamic strain aging effect. Furthermore, the slope of the stress curve of the second deformation is smaller than the slope of the first deformation. The influence of the value of the plastic pre-straining for the second stage behavior was also investigated. For a high plastic pre-straining e.g. $\epsilon>0.1$ one can observe almost a perfectly plastic behavior of the material and the model prediction as well during the second deformation. In other words, for a high plastic

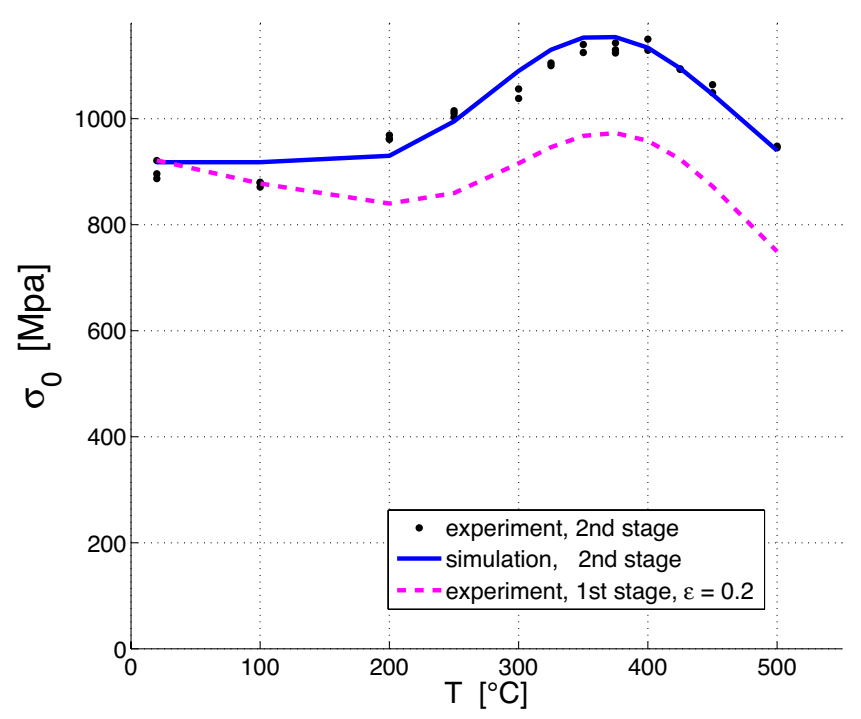

Fig. 6 Yield strength vs. temperature at a pre-deformation of $\epsilon=0.2$ 

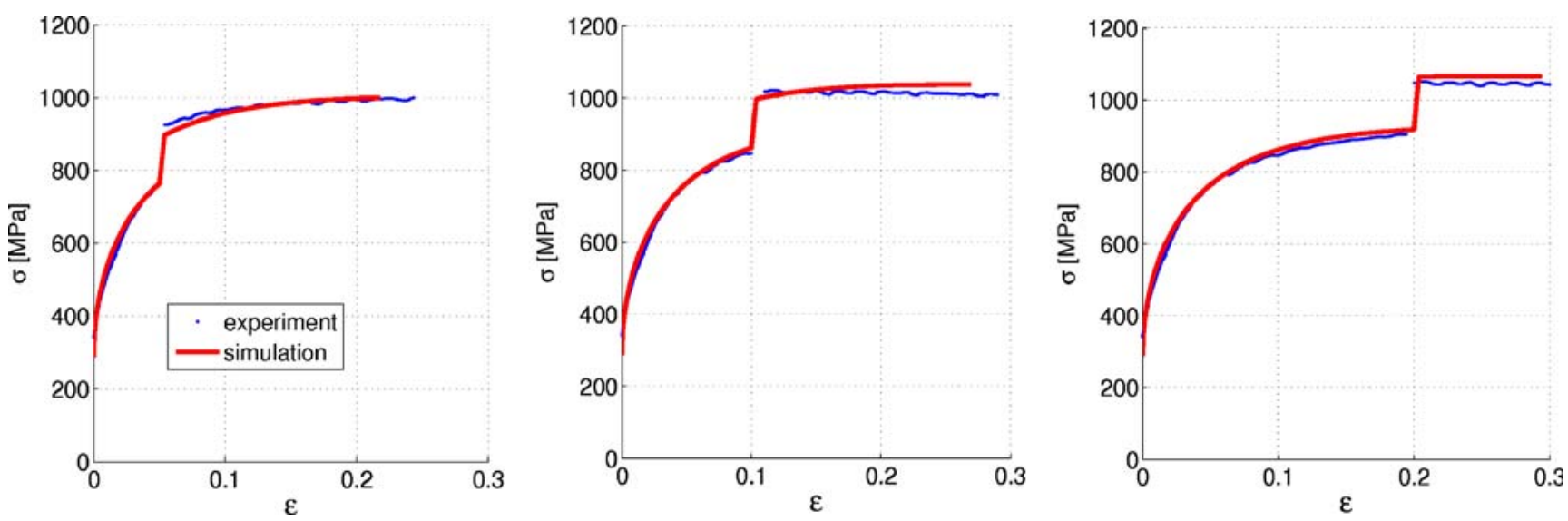

Fig. 7 Two stage deformation: 1st stage at elevated temperature $\left(300^{\circ} \mathrm{C}\right)$, 2nd stage at room temperature

pre-straining the yield strength will increase due to the dynamic strain aging at the expense of a lower ductility.

\section{Conclusions and outlook}

In this work, an enhanced Bergström material model was presented. The simulation results are in good agreement with the experimental data. Especially the temperature dependence of the immobilization rate $B(T)$ is well described. In order to describe the two stage deformation process the evolution equation of the classical Bergström model Eq. 2 was modified. The assumption made in Eq. 10 regarding the splitting of the total dislocation density into free dislocations and locked dislocations provides very good simulation results. The developed model is applicable to a temperature range where dynamic strain aging occurs as well as at room temperature. It is clear that at higher temperatures other phenomena will occur, for example at $T \approx 0.5 T_{m}$, where $T_{m}$ is the melting temperature, recrystallization or other softening mechanisms of the material should be taken into account. The prediction of the yield strength of the second deformation as well as the hardening behavior is in good agreement with the measured data of the compression tests. Only one state variable, the dislocation density, was used to store the deformation history of the pre-deforming process.

In the performed experiments the loading direction between the first and the second deformation was the same. In order to simulate more general deformation processes, more complex experiments are required. Preliminary experiments on cubic specimens showed that different load directions of the first and the second stage cause a different yield strength and hardening behavior of the material, where as discussed in this paper the first deformation is performed at elevated temperatures and the second deformation is performed at room temperature. Besides other phenomena like hardening mechanism and crystal structure the number of active slip systems depends on the stress state and the hardening history (Nemat-Nasser 2004). Above all, experiments with orthogonal strain paths were performed in order to investigate the anisotropy properties of the material. The modeling of such complicated deformation paths which require the mechanics of microbands to take into account is a problem not yet understood (Peeters et al. 2001). The experimental data depicted in Fig. 5 shows that for a higher strain rate the curve shifts, the stress decreases and the region of the dynamic strain aging becomes larger. With the discussed Eq. 13 it is possible to describe the shifting of the curve, but not all the mentioned phenomena. In order to take this into account, further modification of Eq. 5 are necessary. For example, measuring the concentration of the free interstitial atoms is another important task to improve the material model.

Acknowledgements The authors are very grateful to CTI (The Swiss Innovation Promotion Agency) for financial support of this study.

\section{References}

Anon N (1990) Elevated-temperature properties of ferritic steels. In: Steiner R (ed) American society for metals ASM handbook volume 1 properties and selection: irons, steels, and high-performance alloys, 10th edn. ASM International, Materials Park, pp 617-652

Bergstöm Y (1970) A dislocation model for the stress-strain behaviour of polykristalline alpha-Fe with special emphasis on the variation of the densities of mobile and immobile dislocations. Mater Sci Eng 5:193-200 
Bergstöm Y (1983) The plastic deformation of metals. Rev Powder Metal Phys Ceram 2:79-265

Cottrell AH (1953) Dislocations and plastic flow in crystals. Clarendon, Oxford

Gündüz S (2002) Dynamic strain ageing effects in niobium microalloyed steel. Ironmak Steelmak. doi:10.1179/ 030192302225004575

Jiang $\mathrm{H}$ et al (2007) Three types of portevin-le chatelier effects: experiment and modelling. Acta Mater 55:2219-2228

Mecking H, Kocks U F (1981) Kinetics of flow and strainhardening. Acta Metall 29:1865-1875
Nemat-Nasser S (2004) Plasticity. Cambridge University Press, Cambridge

Peeters B et al (2001) Work hardening/softening behaviour of bcc polycrystals during changing strain paths: I. Acta Mater 49:1607-1619

van Liempt $\mathrm{P}$ et al (2002) Modelling the influence of dynamic strain ageing on deformation behaviour. Adv Eng Mater 4(4):225-232

Zeghib NE, Klepaczko JR (1996) Work hardening of mild steel within dynamic strain ageing temperatures. J Mater Sci 31:6085-6088 